http://jmscr.igmpublication.org/home/ ISSN (e)-2347-176x ISSN (p) 2455-0450 crossref DOI: https://dx.doi.org/10.18535/jmscr/v8i8.01

\title{
Prevalence of urinary tract infections among type 2 diabetic patients in Kisii Teaching and Referral Hospital, Kenya
}

\author{
Author \\ Mageto Vincent Mogaka ${ }^{*}$ \\ ${ }^{1}$ International Rescue Committee, Nairobi \\ *Corresponding Author \\ Mageto Vincent Mogaka
}

\begin{abstract}
Introduction: Non-insulin diabetes mellitus is a risk factor for urinary tract infections. Irrational use of antibiotics has led to emergency of uropathogens resistant to available antibiotics. This study sampled diabetic patients to find the prevalence of urinary tract infection among them. The objective of the study was to determine the prevalence of the bacterial agents causing urinary tract infection among diabetic patients.

Method: One hundred and eighty (180) diabetic patients were recruited to take part in the study. Urine samples were collected and cultured for urinary tract infections diagnosis.

Results: Out of the 180 participants, 107 (59.4\%) were male and 73 (40.6\%) were female. The overall prevalence of urinary tract infections was 37 (20\%). The bacterial isolates were Escherichia coli 21 (56.8\%), Klebsiella pneumoniae 10 (27\%), Proteus mirabilis 4 (10.8\%) and Enterococcus faecalis 2 (5.4\%). Prevalence of urinary tract infection was $64.9 \%$ in males and $35.1 \%$ in females.

Discussion: Urinary tract infection is a significant contributor to morbidity among type 2 diabetes mellitus patients. Findings from this study show a high prevalence of infection in males compared to females. Escherichia coli is the predominant isolate causing infection among the participants. The burden of management of this infection is still huge.

Conclusion: More males than females were involved in the study with females recording a lower prevalence of urinary tract infection compared to males.

Keywords: Non-insulin dependent diabetes mellitus, Urinary tract infection.
\end{abstract}

\section{Introduction}

According to the World Health Organization (WHO), the prevalence of diabetes in Kenya was $1.6 \%$ as at $2014^{(1)}$. Diabetic cases are projected to increase due to lifestyle changes. Urinary tract infection is the main cause of morbidity among diabetic patients. This is especially when the UTI is left unattended to. Obesity, gender and prostate syndrome in men have been identified as some of the risk factors for $\mathrm{UTI}^{(2)}$. Diabetes mellitus is associated with bladder dysfunction, glycosuria and low immunity, all of which predispose an individual to UTI. Increasing cases of diabetes mellitus which consequently lead to more UTI cases and irrational use of antibiotics has led to emergence of multi-drug resistant strains. Studies in Africa have shown the need to have systematic screening of UTI in diabetic patients due to the 
increasing prevalence ${ }^{(3,4)}$. In Sudan, symptomatic and asymptomatic bacteriuria among diabetic patients was found to be $17.1 \%$ and $20.9 \%$ respectively $^{(5)}$. The same study showed that there was a higher prevalence of UTI among male diabetic patients compared to female diabetic patients. In another study analysing the challenges of UTI, it was concluded that studies focusing on subgroups of patients who are likely to suffer more frequent infections like diabetic patients would be very useful in the management of $\mathrm{UTI}^{(6)}$. As a developing nation, Kenya is one of the countries bearing the burden of increasing morbidity among type 2 diabetes mellitus patients in Africa. Data on UTI cases among type 2 diabetic patients was missing in over $42.6 \%$ countries of the African continent ${ }^{(3)}$. This study was conducted at the Kisii teaching and Referral Hospital, Kenya, to provide an up to date status on the prevalence of UTI among type 2 diabetics. The facility serves patients from over five counties in the region thus providing a good sample population for the study.

\section{Methods and Methods}

The study was conducted in Kisii teaching and Referral Hospital in Kisii County. The referral facility serves patients from south Nyanza, rift valley and western counties in the country. Western Kenya and Nyanza counties are highly populated and this provided a high population demanding quality healthcare services in the region. A cross-sectional study was adopted among non-insulin dependent diabetes mellitus patients visiting Kisii teaching and Referral Hospital in Kisii County. The study comprised of non-insulin dependent diabetes mellitus patients visiting Kisii Teaching and Referral Hospital in Kisii County. The participants were outpatient patients only visiting the diabetes clinic in the facility. Patients who have not been on antibiotic therapy for at least two weeks were eligible for the study. Non-insulin diabetes mellitus patients were sampled from the diabetic outpatient clinic. Patients aged over 25 years of age were selected as the study looked to focus on type 2 diabetes mellitus which is commonly adult onset. Patients on antibiotic therapy for at least two weeks were not eligible for the study as this would affect the sensitivity and isolation of bacterial pathogens from their urine samples. Every fourth patient visiting the clinic was selected to take part in the study. Only patients who signed the consent forms were allowed to take part in the study.

\section{Ethical Clearance}

This study was carried out in accordance to the declaration of Helsinki and International Conference on Harmonization Guideline on Good Clinical Practice (ICH-GCP). The protocol and informed consent form were reviewed and approved by the Kenyatta University Ethics Review Committee (PKU/610/I694 on $18^{\text {th }}$ May 2017). The research permit was granted by National Commission for Science, Technology and Innovation (NACOSTI/P/17/88572/17706 ON $6^{\text {th }}$ July 2017) Kenya. Written informed consent was then obtained from each participant. A unique identification code was assigned to every participant to ensure confidentiality of the findings. All records were stored in a locked cabinet in a secure room accessible to the principal investigator.

\section{Laboratory Procedure}

\section{Sample Collection and Processing}

A total of 180 urine samples were collected from the participants using the clean catch mid-stream morning technique in sterile containers ${ }^{(7)}$. Each and every participant was asked to fill in a pretested questionnaire before a urine sample was collected. As soon as the samples were collected, they were labelled and transported to the laboratory for analysis. About $20 \mathrm{ml}$ of urine was collected in every participant. All urine samples were analyzed in the Kisii Teaching and Referral Hospital microbiology laboratory.

With the help of a standard quantitative wire loop, all samples were inoculated onto cysteine lactose electrolyte deficient agar. The plates were 
incubated at 37 degrees Celsius for 24 hours. The resultant outcome was classified as significant, non-significant or contaminated. Contaminated samples were discarded. In line with the WHO definition of UTI, culture plates showing $\geq 10^{5}$ colony forming units per $\mathrm{mL}$ of a single bacterial species were considered significant ${ }^{(8)}$. Bacteriuria in participants who presented with symptoms of UTI was symptomatic bacteriuria while in those who did not present with symptoms of UTI it was asymptomatic bacteriuria.

\section{Identification of species and antibiotic susceptibility testing}

Isolates from all significant culture results were gram-stained. Biochemical tests were then carried out to confirm the species of the isolates. E. coli was confirmed by its pink colonies and a positive indole test whereas $K$. pneumoniae was confirmed by pink colonies with a negative oxidase and indole test. Proteus mirabilis was confirmed by small pale to colourless colonies testing positive for indole and urease but negative to oxidase. Enterococcus faecalis was the only gram positive isolate. It was confirmed by small coccoid colonies testing negative to catalase and citrate test. Frequency tables were used to determine the prevalence of urinary infection among participants.

\section{Results}

Thirty seven participants out of the one hundred and eighty that took part in the study tested positive for urinary tract infection. The prevalence of UTI was found to be at $20.6 \%$. According to the findings, $64.9 \%$ (24 patients) were males compared to $35.1 \%$ (13 patients) who were females. A further comparison based on gender revealed that $22.4 \%$ of male participants against $17.8 \%$ of female participants tested positive for UTI. Gender revealed no statistical significance in terms of positive UTI testing $(\mathrm{p}=0.45)$. Male participants were, however, found to be 1.335 (odds ratio) times more likely to test positive for UTI that their female counterparts.
Table 1: Prevalence of bacterial pathogens causing UTI among male and female participants

\begin{tabular}{|l|cc|cc|}
\cline { 2 - 4 } \multicolumn{1}{c|}{} & MALE $(\%)$ & \multicolumn{2}{c|}{ FEMALE $(\%)$} \\
\hline E. coli $(\mathrm{n}=21)$ & $10(27.0)$ & 11 & $(29.7)$ \\
K. pneumoniae $(\mathrm{n}=10)$ & 9 & $(24.3)$ & 1 & $(2.7)$ \\
$P$. mirabilis $(\mathrm{n}=4)$ & 4 & $(10.8)$ & 0 & $(0.0)$ \\
E. faecalis $(\mathrm{n}=2)$ & 1 & $(2.7)$ & 1 & $(2.7)$ \\
\hline
\end{tabular}

As shown in Table 1 above; Escherichia coli was responsible for $56.8 \%$ of the total UTI cases in this study. Out of the $21 \mathrm{E}$. coli isolates, 10 $(47.6 \%)$ were isolated from male participants while $11(52.4 \%)$ were from female participants. Klebsiella pneumoniae was responsible for $27.0 \%$ of the total UTI cases in the study. Out of the 10 K. pneumoniae isolates, 9 (90\%) were from males and $1(10 \%)$ from females. Proteus mirabilis constituted $10.8 \%$ of the total UTI cases in the study. All the $4(100 \%)$ P. mirabilis isolates were from male participants. Enterococcus faecalis constituted $5.4 \%$ of the total UTI cases in the study. One $(50 \%)$ isolate was each obtained from male and female participants.

\section{Discussion}

Male participants were found to have a higher prevalence of UTI $(64.9 \%)$ compared to their female counterparts $(35.1 \%)$ similarly to what was found in Sudan ${ }^{(5)}$. Contrary the finding of this study where males had a higher prevalence of UTI than females, a study in the United Arab Emirates and Romania found a higher frequency of UTI in females than in males ${ }^{(9,10)}$. The general perception is that due to the short urethra in females, they are predisposed to getting UTI more than males. The findings in this study, however, give a different opinion. It is possible that extensive public health education has improved on hygienic practices in women and hence reduced cases of UTI.

Escherichia coli have been found to be a predominant isolate in urinary tract infection both in male and females. According to studies carried out in Sudan, Israel and India E. coli was found to be the leading isolate among type 2 diabetes mellitus patients ${ }^{(5,11-13)}$. This finding is in agreement with what was found in this study. The 
prevalence of $E$. coli among type 2 diabetic patients in a study carried out in 2011 was found to be at $33.3 \%$ in Ethiopia and $29.6 \%$ in India ${ }^{(4}$ 11). In 2015, a similar study in Sudan found the prevalence of $E$. coli among diabetic patients to be $56.4 \%{ }^{(5)}$. This study which was carried out in Western Kenya found the prevalence of E. coli among diabetic patients to be $56.8 \%$. There is a significant increase in the prevalence of $E$. coli over the years going by the findings of the above mentioned studies. The increased prevalence could be attributed to low turnout for medical attention or antibiotic resistance.

The same case of increasing prevalence was also noted in K. pneumoniae from $19 \%$ in $2011^{(4)}$, then $23 \%$ in $2015^{(5)}$ and now $27 \%$ in this study which was conducted in 2017. In one study carried out in Nepal, K. pneumoniae was found to be the least isolate after E. coli, Staphylococcus aureus and Enterococcus species ${ }^{(14) .}$ Studies have found out that age and gender of a given population also contribute to the increasing prevalence of UTI among type 2 diabetic patients $(15,16)$. It is evident that prevalence of UTI among diabetic patients is on the rise. Other bacterial pathogens like E. faecalis and P. mirabilis could be hospital acquired or signify low immunity in the affected individuals.

\section{Conclusion}

Prevalence of UTI among diabetic patients is a worrying trend that is on the rise. It is imperative for surveillance and more studies to be carried out so that clinicians can be equipped with up to date information that will help them in the management of UTI. Urinary tract infection is a major cause of morbidity in diabetes patients and contributes to the burden of health care delivery.

\section{References}

1. World Health Organization. Diabetes. June 21, 2017. http://www.who.int/mediacentre/factsheets/f s312/en/. Accessed $5^{\text {th }}$ February 2018.
2. Sewify M, Nair S, Warsame S, Murad M, Alhubail A, Behbehani K, Al-Refaei F, Tiss A. Prevalence of Urinary Tract Infection and Antimicrobial Susceptibility among Diabetic Patients with Controlled and Uncontrolled Glycemia in Kuwait. Journal of Diabetes Research. 2015: 1-7.

3. Tadesse BT, Ashley AE, Ongarello S, Havumaki J, Wijegoonewardena M, Gonzalez IJ, Dittrich S. Antimicrobial resistance in Africa: a systematic review. BMC Infectious Diseases, 2017: 17: 616.

4. Beyene G, Tsegaye W. Bacterial uropathogens in urinary tract infection and antibiotic susceptibility pattern in Jimma University Specialized Hospital, Southwest Ethiopia. Ethiop J Health Sci. 2011: 21(2): 141-146.

5. Hamdan ZH, Kubarr E, Adam AM, Hassan OS, Suliman OS, Adam I. Urinary tract infections and antimicrobial sensitivity among diabetic patients at Khartoum, Sudan. Annals of Clinical Microbiology and Antimicrobials. 2015: 14-26.

6. Stamm WE, Norrby SR. Urinary Tract Infections: Disease Panorama and Challenges. The Journal of Infectious Diseases. 2001: S1-S4.

7. Cheesbrough M, District Laboratory Practice in Tropical Countries. London: Cambridge University Press, 2010: 106-115

8. Nigussie D, Amsalu A. Prevalence of uropathogen and their antibiotic resistance pattern among diabetic patients. Turk $J$ Urol. 2017: 43 (1): 85-92.

9. Mubarak AA, Ashraf AM, El-hag M, Raza MA, Majed A, Al-Sharbatti S, Shaikh RB, Khan FA. Prevalence of urinary tract infections among diabetes mellitus and nondiabetic patients attending a teaching hospital in Ajman, UAE. Gulf Medical Journal. 2012: 228-232.

10. Chita T, Licker M, Sima A, Vlad A, Timar B, Sabo P, Timar R. Prevalence of Urinary Tract Infections in Diabetic Patients. Rom J 
Diabetes Nutr Metab Dis. 2013: 20(2): 99105.

11. Nitzan O, Mazen E, Chazan B, Saliba W. Urinary tract infections in patients with type 2 diabetes mellitus: review of prevalence, diagnosis, and management. Diabetes, Metabolic Syndrome and Obesity: Targets and Therapy. 2015: 8: 129-136.

12. Saleem M, Daniel B. Prevalence of Urinary Tract Infection among Patients with Diabetes in Bangalore City. International Journal of Emerging Sciences. 2011: 2(1): 133-142.

13. Shill MC, Huda NH, Karmakar UK, Moain, FB. Prevalence of Uropathogens in Diabetic Patients and Their Corresponding Resistance Pattern: Results of a Survey Conducted at Diagnostic Centres in Dhaka, Bangladesh. Oman Medical Journal. 2010: 25(4): 282-286.

14. Jha PK, Baral R, Khanal B. Prevalence of Uropathogens in Diabetic Patients and Their Susceptibility Pattern at a Tertiary Care Center in Nepal-A Retrospective Study. International Journal of Biomedical Laboratory Science (IJBLS). 2014: 3(2): 2934.

15. Hirji I, Guo Z, Andersson SW, Hammar N, Gomez-Caminero A. Incidence of urinary tract infection among patients with type 2 diabetes in the UK General Practice Research Database (GPRD). Journal of Diabetes and Its Complications. 2012: 26: 513-516.

16. Hammar N, Farahmand B, Gran M, Joelson $\mathrm{S}$, Anderson, SW. Incidence of urinary tract infection in patients with type 2 diabetes. Experience from diverse event reporting in clinical trials. Pharmacoepidemiology and drug safety. 2010: 19: 1287-1292. 